\title{
Non-volatile fatty acids in the diagnosis of non-specific vaginitis
}

\author{
CA ISON*, CSF EASMON*, SG DAWSON*, G SOUTHERTON*, JWR HARRIS \\ From the *Department of Medical Microbiology, Wright-Fleming Institute, St Mary's Hospital Medical \\ School, and the Department of Genitourinary Medicine, St Mary's Hospital, London W2 1PG
}

SUMMARY In the vaginal washings of 100 women with symptomatic non-specific vaginitis a succinate/lactate ratio of $\geqslant 0.4$ had a diagnostic sensitivity of $80 \%$, a specificity of $83 \%$ for this condition. The predictive value of a positive test was $94 \%$, but that of a negative test was only $55 \%$. A strong association between the presence of Gardnerella vaginalis, anaerobes, a vaginal $\mathrm{pH}$ of above $4 \cdot 5$, and amines was found not only in non-specific vaginitis, but also in trichomonal and gonococcal infection. A variety of primary changes may encourage the multiplication of both gardnerellae and anaerobes and their presence in non-specific vaginitis may be a secondary rather than a primary event.

The aetiology of non-specific vaginitis is still a matter for debate. $G$ vaginalis and Bacteroides $s p$, particularly of the oralis-melaninogenicus group, have been implicated either alone or in combination. The association with anaerobes and the absence of neutrophils in the discharge have led to the use of the term "anaerobic vaginosis".

Non-specific vaginitis is usually diagnosed clinically by the presence of an increased vaginal discharge with few polymorphs in the absence of any recognised pathogen. The discharge is homogeneous in nature with a $\mathrm{pH}$ of $>4.5$. "Clue cells" are present and a "fishy" odour is produced on the addition of $10 \%$ potassium hydroxide to the washings. ${ }^{2}$ Spiegel $e t a l^{2}$ have suggested that the presence of two out of four criteria ( $\mathrm{pH}>4.5$, homogeneous discharge, "clue cells," amines) should be taken to indicate non-specific vaginitis. All these criteria are, however, subjective and a simple, more objective rapid test to aid clinical diagnosis would be useful.

The analysis of non-volatile fatty acids in vaginal washings as described by Spiegel $e t a^{2}$ introduced the possibility of such a technique. They showed that while lactate was the predominant non-volatile fatty acid present in normal vaginal washings, succinate was increased in patients with non-specific vaginitis diagnosed on the basis of their own four criteria (see above). A succinate/lactate ( $\mathrm{S} / \mathrm{L})$ ratio of $>0.4$ gave a $90 \%$ positive predictive value for non-specific vaginitis in these patients.

Accepted for publication 19 July 1983
We have attempted to evaluate non-volatile fatty acid analysis as a diagnostic test for non-specific vaginitis by testing vaginal washings from asymptomatic women, those with trichomonal and candida vaginitis, with gonorrhoea and with non-specific vaginitis. We did not, however, use Spiegel's criteria.

\section{Material and methods}

\section{PATIENT SELECTION}

A total of 251 women attending the sexually transmitted diseases (STD) clinic, who had not received antibiotic therapy in the preceding two weeks, were included. A control group consisted of 29 asymptomatic women with a normal vaginal discharge. Symptomatic women with a discharge fell into four groups. Fifty-four women had trichomonal vaginitis, 51 candidal infection, both diagnosed on microscopy, while 17 had gonorrhoea diagnosed by culture. None of these women had mixed infections. In the remaining 100 patients no specific pathogen was found (all had "clue cells") and these were considered to have non-specific vaginitis. In defining this non-specific vaginitis group we did not use Spiegel's four criteria (raised vaginal pH, amines, "clue cells", homogeneous discharge).

\section{SPECIMEN COLLECTION}

Each patient had a vaginal examination during which the following specimens were collected in the order described.

After insertion of a speculum a cervical swab was 
taken and immediately placed on neisseria isolation medium for $N$ gonorrhoeae. A plastic disposable loop (Gibco) was then inserted into the vagina and discharge collected firstly for microscopical examination for Trichomonas vaginalis, candida and "clue cells", and secondly for $\mathrm{pH}$ estimation using narrow range $\mathrm{pH}$ paper 4-6 $(\mathrm{BDH})$. Isotonic saline $(2 \mathrm{ml})$ was introduced through the speculum and pooled using a swab, which was then placed in Amies medium for transfer to the laboratory for aerobic and anaerobic culture. The saline was aspirated by a syringe into a sterile universal (Sterilin). Specimens were not taken for isolation of either Chlamydia or Mycoplasma sp. All specimens were processed in the laboratory within four hours.

\section{MICROSCOPY}

Wet preparations were examined by one person (SGD) in the STD clinic, using a $\times 40$ objective. The presence of $T$ vaginalis, "clue cells" and candida was noted. Smears were made from the deposit of the vaginal washings and examined using Gram's stain for "clue cells" and Gram-variable bacilli.

\section{CULTURAL METHOD}

$N$ gonorrhoeae was isolated on neisseria isolation medium containing GC agar base Difco (36 g/l) + $1 \%$ Isovitalex (BBL) made selective by the addition of vancomycin, colistin, trimethoprim and amphotericin. Swabs were inoculated directly in the STD clinic, and stored before and after transfer to the laboratory in $7 \% \mathrm{CO}_{2}$ at $36^{\circ} \mathrm{C}$ for $48 \mathrm{~h} . \mathrm{N}$ gonorrhoeae isolates were identified as oxidase positive Gram-negative cocci, that utilised glucose but not maltose, lactose and sucrose. $G$ vaginalis was isolated on bilayer blood agar, using $5 \%$ outdated human banked blood in the top layer made selective by the addition of gentamicin, nalidixic acid and amphotericin as previously described ${ }^{3}$ and incubated at $36^{\circ} \mathrm{C}$ in $7 \% \mathrm{CO}_{2}$ for $48 \mathrm{~h}$. Gram-variable bacilli that showed $\beta$-haemolysis on human but not on horse blood agar and were catalase- and oxidasenegative were identified as $G$ vaginalis. Previous unpublished data (Ison) showed that bacteria fulfilling these criteria produced acid from starch and maltose.

Other aerobic organisms were cultured on $5 \%$ horse blood for $24 \mathrm{~h}$ at $36^{\circ} \mathrm{C}$ in $7 \% \mathrm{CO}_{2}$ and noted only if large numbers present. All obligate anaerobes were isolated on enriched blood agar, consisting of reinforced clostridial base agar (Oxoid), $52.5 \mathrm{~g} / \mathrm{l}$; liver digest (Oxoid), $10 \mathrm{~g} / 1$ and $5 \%$ horse blood. This base was used both as a nonselective medium and selective by the addition of $\vec{\circ}$ kanamycin, $100 \mathrm{mg} / \mathrm{l}$, and vancomycin, $75 \mathrm{mg} / \mathrm{l}$. All cultures were incubated at $37^{\circ} \mathrm{C}$ in an anaerobic $\stackrel{\omega}{\sigma}$ cabinet (Don Whitley) for five days. Initially, obli- 옹 gate anaerobes were identified as being sensitive to metronidazole, $(5 \mu \mathrm{g}$ disc). Further identification has been carried out but is not included in this part $\vec{N}$ of the study.

\section{PRODUCTION OF AMINES}

A drop from the vaginal washing was mixed on a glass slide with an equal volume of $10 \%$ potassium $\Phi$ hydroxide $(\mathrm{BDH})$. This neutralises the discharge and produces a "fishy odour" thought to be due to release of amines.

\section{ANALYSIS OF NON-VOLATILE FATTY ACIDS}

\section{After centrifugation of the vaginal washing} deposit epithelial and/or pus cells, the supernatant was acidified, mixed with methanol and the methyl esters extracted by chloroform as described by Spiegel et al. ${ }^{2}$ A $1 \mu$ l volume was injected onto a $\stackrel{\square}{\unrhd}$ column packed with $10 \% \mathrm{SP} 1000 / 1 \% \mathrm{H}_{3} \mathrm{PO}_{4}$ on $\vec{F}$ 100/200 Chromosorb (Supelco). The column temperature was $170^{\circ} \mathrm{C}$ and the nitrogen carrier gas, 60 $\mathrm{ml} / \mathrm{min}$. A series 104 gas chromatograph (Pye Unicam) was used, and non-volatile fatty acid standards included pyruvic, oxalacetic, oxalic, methyl, ? malonic, fumaric and succinic (Radley). The ratio of $\frac{5}{3}$ the succinate peak to that of the lactate was then calculated (S/L ratio). The sensitivity, specificity and 0 the predictive value of an $\mathrm{S} / \mathrm{L}$ ratio of $\geqslant 0.4$ (posi-

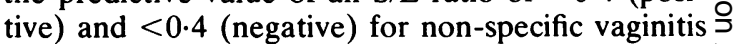
were calculated as follows:

$$
\begin{aligned}
& \text { Sensitivity }=\frac{\text { No women with non-specific vaginitis with } \mathrm{S} / \mathrm{L} \text { ratio } \geqslant 0.4}{\text { Total No women with non-specific vaginitis tested }} \\
& \text { Specificity }=\frac{\text { No women without non-specific vaginitis with an } \mathrm{S} / \mathrm{L} \text { ratio }<0.4}{\text { Total No women without non-specific vaginitis tested }} \\
& \text { Predictive value of positive test }=\frac{\text { No } \mathrm{S} / \mathrm{L} \text { ratios } \geqslant 0.4 \text { in women with non-specific vaginitis }}{\text { Total No } \mathrm{S} / \mathrm{L} \text { ratios } \geqslant 0.4}
\end{aligned}
$$

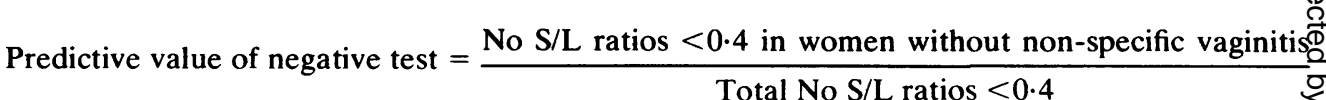




\section{Results}

All patients entering this study with symptomatic vaginitis, not caused by $T$ vaginalis, $N$ gonorrhoeae or Candida spp, were found to conform to two or more of the four criteria suggested by Spiegel $e a^{2}$ as being suggestive of non-specific vaginitis. Four of 29 asymptomatic controls had similar findings.

The distribution of $\mathrm{S} / \mathrm{L}$ ratios in vaginal washings from patients with non-specific vaginitis and controls is shown in the Figure. In our hands this test had a sensitivity of $80 \%$ and a specificity of $83 \%$ while the predictive value of a positive test was $94 \%$. However, as 20 out of the 100 symptomatic patients had low $\mathrm{S} / \mathrm{L}$ ratios, the predictive value of a negative test was only $55 \%$. Clue cells were found in vaginal samples from all 20 of these patients and they all had at least two of the four criteria for nonspecific vaginitis already mentioned. $G$ vaginalis and anaerobes were isolated from $13 / 20$, anaerobes alone from $3 / 20$ and $G$ vaginalis alone from $4 / 20$. Five asysmptomatic women in our control group had a raised S/L ratio. Four of these, however, despite their lack of symptoms would have been diagnosed as having non-specific vaginitis on Spiegel's criteria and on culture had both gardnerella and anaerobes. The fifth had clue cells, but nothing else.

Both $G$ vaginalis and anaerobes have been implicated as the aetiological agent of non-specific vaginitis. The presence of these bacteria together with the release of amines and a raised $\mathrm{pH}$ was monitored in all patients (Table). The isolation of $G$ vaginalis and anaerobes was high not only in NSV, but also in trichomonal vaginitis and gonorrhoea. This close association, which was also true of raised vaginal $\mathrm{pH}$ and the presence of amines, was not seen either in asymptomatic controls or in women with candida vaginitis.

The isolation rate of $G$ vaginalis and anaerobes in the controls is high when compared with that found by others. ${ }^{4}$ This probably reflects differences in either patient selection or in measurement. We used qualitative culture with selective media rather than quantitative counts.

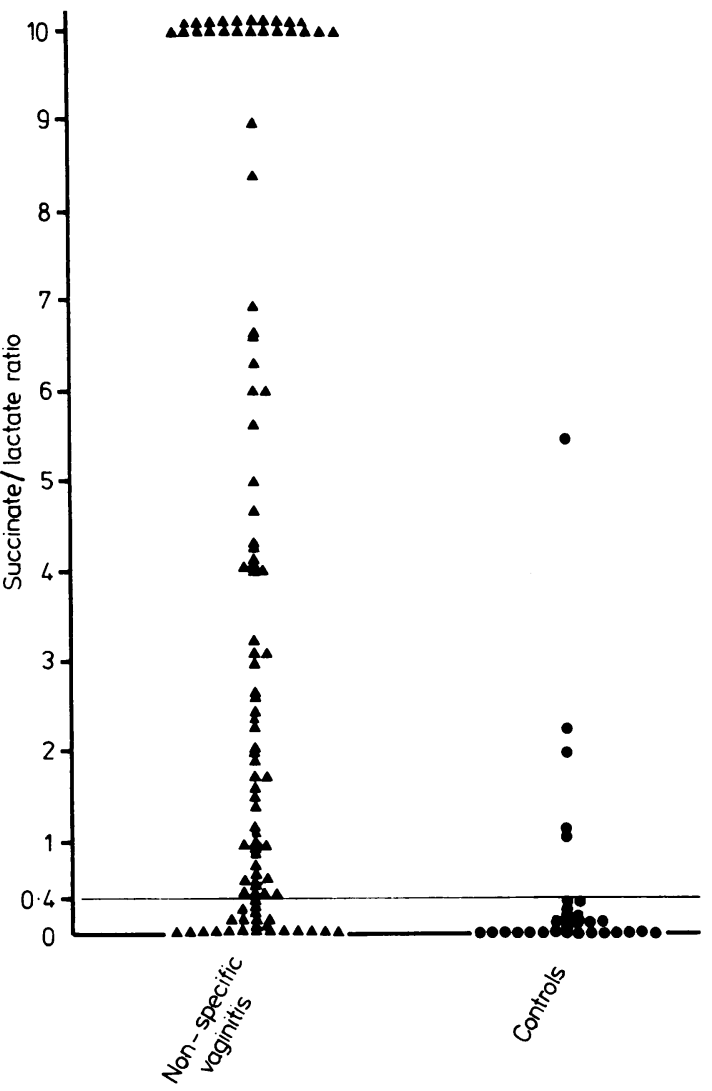

Succinatellactate ratios in the vaginal washings of asymptomatic women and those with non-specific vaginitis.

\section{Discussion}

In terms of sensitivity, specificity and the predictive value of a positive test, our results compare well with Spiegel's original description of the test. ${ }^{2}$ However, we found the predictive value of a negative test to be much lower as $20 \%$ of our non-specific vaginitis patients had $\mathrm{S} / \mathrm{L}$ ratios below $0 \cdot 4$. Piot et al ${ }^{\mathrm{s}}$ also evaluated this technique. Using a succinate lac-

Relation of increased succinate/lactate ratio to presence of Gardnerella vaginalis and/or anaerobes

\begin{tabular}{lllllll}
\hline $\begin{array}{l}\text { Cause of } \\
\text { vaginitis }\end{array}$ & $\begin{array}{l}\text { Total } \\
\text { tested }\end{array}$ & \% positive & & & \\
\cline { 2 - 7 } & & $S / L>0.4$ & $G$ vaginalis & Anaerobes & Amines & $p H>4.5$ \\
\hline $\begin{array}{l}\text { Non-specific } \\
\text { Trichomonas }\end{array}$ & 100 & 78 & 90 & 92 & 85 & 68 \\
vaginalis & 54 & 69 & 93 & 83 & 80 & 63 \\
Gonorrhoea & 17 & 82 & 55 & 48 & 83 & 12 \\
Candida & 51 & 14 & 35 & 61 & 31 & 6 \\
Controls & 29 & 14 & &
\end{tabular}


tate ratio of 0.3 rather than 0.4 , they found that $75 \%$ of women with non-specific vaginitis had a raised ratio as compared with $10 \%$ of controls. The importance of patient selection and clinical definition of non-specific vaginitis has been stressed recently and much of the controversy surrounding this topic probably relates to differences of definition and selection. ${ }^{46}$ Our 100 NSV patients were selected primarily on the basis of symptoms, while Spiegel et al ${ }^{2}$ ignored symptoms and defined non-specific vaginitis on the basis of two or more of the following four criteria: a pH of above 4.5, a typical homogeneous discharge, clue cells and a characteristic fishy odour released from vaginal washings on the addition of potassium hydroxide. In fact all our 100 non-specific vaginitis patients fulfilled these criteria. Patient selection alone cannot, therefore, account for the low succinate/lactate ratios found in 20 of the 100,16 of whom had significant numbers of anaerobes present in vaginal samples. Seventeen of the 20 had $G$ vaginalis while 13 had both $G$ vaginalis and anaerobes. In our control group of 29, patient selection played a more significant role. Four of the five controls with a raised succinate/lactate ratio would have been diagnosed as NSV on the basis of Spiegel's criteria. We would, therefore support the use of these criteria for the diagnosis of non-specific vaginitis rather than relying on the presence or absence of symptoms. Recently the same group in Seattle ${ }^{6}$ have reviewed this problem and commented on the limitations of primary reliance on symptoms.

The diagnosis of non-specific vaginitis depends on the exclusion of other recognised pathogens. While this is relatively simple with trichomonal and candida infections, we have shown that $82 \%$ of women with gonorrhoea had a raised succinate lactate ratio associated with the presence of gardnerella, anaerobes, the presence of amines, and a raised vaginal $\mathrm{pH}$.

Gonorrhoea in women can be difficult to diagnose and the only generally available rapid test, microscopy, is very insensitive. The use of the succinate/ lactate ratio as a rapid test to supplement or replace existing tests for non-specific vaginitis would not avoid the potential misdiagnosis of a case of gonorrhoea. It would in fact be interesting to know how many women with gonorrhoea are initially diagnosed as having non-specific vaginitis.
The similarity between the findings in trichomonal infection and gardnerella associated vaginitis which we found were also commented upon by Taylor $e t$ $\mathrm{al}^{4}{ }^{4}$ The association between gonorrhoea, anaerobes and gardnerella is more surprising. Balsdon has reported an association both between $G$ vaginalis associated vaginitis and gonorrhoea and the sexual partners of men with non-specific urethritis. ${ }^{7}$ We now plan to examine the relation between $G$ vaginalis and anaerobes and mycoplasma and chlamydia. $G$ vaginalis in low numbers is part of the normal vaginal flora in many women together with anaerobes. ${ }^{6}$ A variety of primary stimuli, such as trichomonal and gonococcal infection, which alter the normal ecology of the vagina and result in a raised $\mathrm{pH}$, encourage the growth of $G$ vaginalis and anaerobes. In non-specific vaginitis the same pattern occurs, but with no obvious initial microbial stimulus. It may be that in non-specific vaginitis neither $G$ vaginalis nor anaerobes play a primary pathogenic role, but merely take advantage of vaginal conditions changed by an unknown stimulus.

We thank the Medical Research Council and May \& $\underset{\Phi}{\overparen{D}}$ Baker Ltd for financial support.

\section{References}

' Blackwell A, Barlow D. Clinic diagnosis of anaerobic diagnosis of anaerobic vaginosis (non-specific vaginitis). A practical guide. Br J Vener Dis 1982;58:387-93.

${ }^{2}$ Spiegel Carol A, Amsel R, Eschenbach D, Schoenknecht F, Holmes KK. Anaerobic bacteria in non-specific vaginitis. $N$ Engl J Med 1980;303:601-7.

${ }^{3}$ Ison CA, Dawson SG, Hilton J, Csonka GW, Easmon CSF. Comparison of culture and microscopy in the diagnosis of Gardnerella vaginalis in fection. J Clin Pathol 1982;35:550-4.

4 Taylor E, Blackwell A, Barlow D, Phillips I. Gardnerella vaginalis, anaerobes and vaginal discharge. Lancet 1982;i:1376-9.

${ }^{5}$ Piot P, Van Dyck E, Godts P, Vanderheyden J. The vaginal microbial flora in non-specific vaginitis. Eur J Clin Microbiol 1982;1:301-6.

- Amsel R, Totten PA, Spiegel CA, Chen KCS, Eschenbach D, Holmes KK. Non-specific vaginitis. Diagnostic criteria and microbial and epidemiologic associations. $A m J M e d$ 1983;74:14-22.

' Balsdon MJ. Gardnerella vaginalis and its clinical syndrome. Eur J Clin Microbiol 1982;1:288-93.

Requests for reprints to: Dr CSF Easmon, Department of $\mathcal{N}$ Bacteriology, St Mary's Hospital Medical School, Wright-Fleming Institute, Paddington, London W2 1PG, England. 